\title{
Emergency oxygen therapy in the dental practice: administration and management
}

- Stresses responsibilities of dentists regarding storage and inspection of oxygen in the dental practice.

- Highlights indications for oxygen therapy in the dental practice.

- Discusses principles of safe oxygen therapy.

P. Jevon'

Dental practitioners should understand when and how to safely and effectively administer emergency oxygen therapy. This article provides an overview on its administration and management, as well as reviewing the hazards associated with its use and storage.

\section{INTRODUCTION}

Dental practices should have facilities to administer high flow oxygen to a patient who is critically ill. ${ }^{1}$ Although oxygen can save lives by preventing severe hypoxaemia, if it isn't administered and managed appropriately, there is a potential for serious harm and even death. ${ }^{2}$

The aim of this article is to provide an overview to the administration and management of emergency oxygen in the dental practice.

\section{RELATED ANATOMY AND PHYSIOLOGY}

Oxygen is a colourless, odourless gas that forms about $21 \%$ of the earth's atmosphere and is essential for plant and human life. Tissue oxygenation is dependent upon inspired oxygen, the concentration of haemoglobin and its ability to saturate with oxygen, as well as the circulation of blood.

\section{EVIDENCE OF HARM ASSOCIATED WITH OXYGEN THERAPY}

Up to June 2009, the National Patient Safety Agency (NPSA) had received 281 reports of serious incidents relating to inappropriate administration and management of oxygen; of these incidents, poor oxygen management appears to have caused nine patient deaths and may have contributed to a further 35 deaths. ${ }^{2}$ Common themes identified from the review of these incidents, local investigations and other sources are:

\section{${ }^{1}$ Resuscitation Officer/Clinical Skills Lead, Mano} Hospital, Walsall

Correspondence to: Phil Jevon

Email:phil.jevon@walsallhealthcare.nhs.uk

\section{Refereed Paper}

Accepted 25 September 2013

DOI: $10.1038 /$ sj.bdj.2014.49

${ }^{\circ}$ British Dental Journal 2014; 216: 113-115

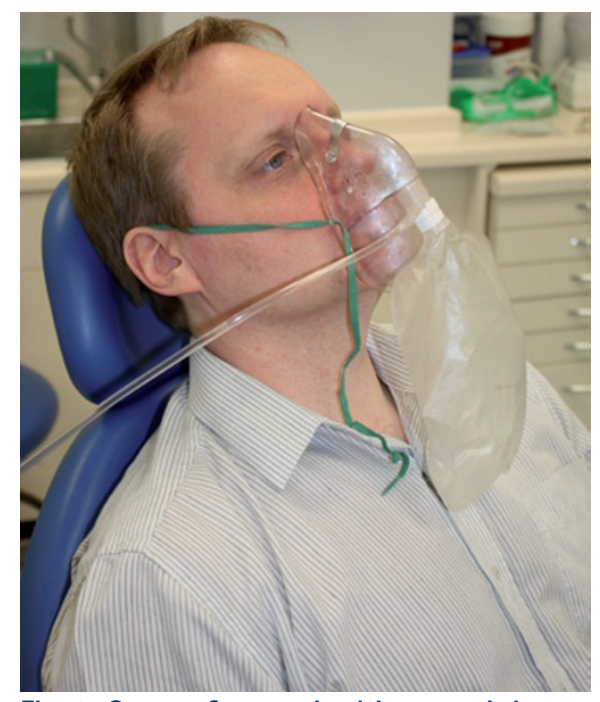

Fig. 1 0xygen face mask with reservoir bag and tubing

- Prescribing: failure to or wrongly prescribed

- Monitoring: patients not monitored, abnormal oxygen saturation levels not acted upon

- Administration: confusion of oxygen with medical compressed air, incorrect flow rates, inadvertent disconnection of supply

- Equipment: empty cylinders, faulty and missing equipment. ${ }^{2}$

Dental practitioners must therefore understand when and how to safely and effectively administer oxygen. An understanding of the hazards associated with the use and storage of oxygen is also important. $^{3}$

\section{INDICATIONS FOR OXYGEN THERAPY IN THE DENTAL PRACTICE}

Oxygen is prescribed to treat hypoxaemia. ${ }^{4}$ Oxygen therapy is therefore aimed at supplementing the inspired oxygen

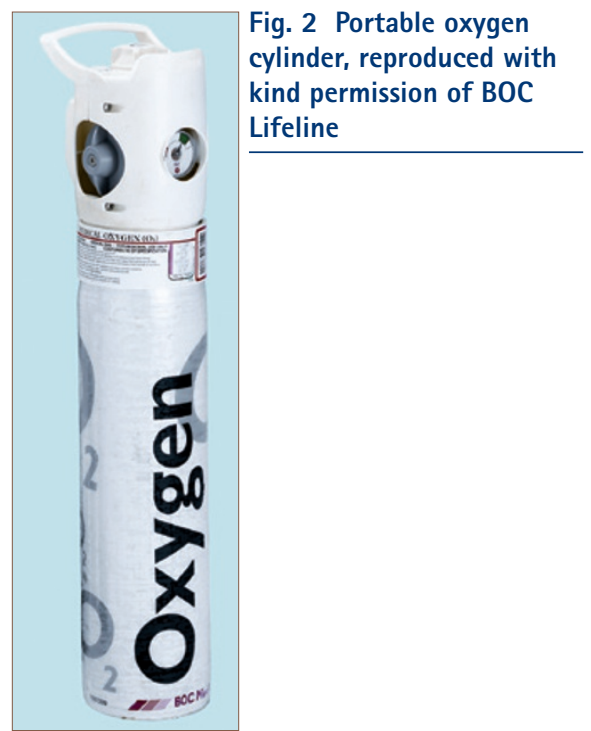

concentration to prevent tissue hypoxia and resultant cellular dysfunction that can occur in the acutely ill patient. ${ }^{5}$

Indications for administration of emergency oxygen in the dental practice include:

- Syncope

- Acute asthma attack

- Anaphylaxis

- During an epileptic fit

- Cardiopulmonary resuscitation. ${ }^{1}$

It is no longer recommended to routinely administer oxygen to a patient with chest pain who is suspected of having a myocardial infarction. High flow oxygen may be administered (15 litres per minute) if the patient is cyanosed (blue lips) or if the level of consciousness deteriorates. ${ }^{1}$

\section{OXYGEN THERAPY EQUIPMENT}

The Resuscitation Council (UK) ${ }^{1}$ recommends that, as a minimum, each dental practice should have: 
- An oxygen face mask with oxygen reservoir and tubing (Fig. 1)

- A portable oxygen cylinder (Fig. 2) of sufficient size to enable the delivery of adequate flow rates, for example, 15 litres per minute, until the arrival of an ambulance or the patient fully recovers. A full 'D' size cylinder contains 340 litres of oxygen and will allow a flow rate of 10 litres per minute for up to 30 minutes. It may be necessary to have two such cylinders in the dental practice to ensure the supply of oxygen does not run out during a medical emergency

- An oxygen cylinder key (if appropriate). Some oxygen cylinders require a special key to unlock the cylinder - it is most important that the key is kept with the cylinder

- Ideally, the patent's oxygen saturation levels should be monitored before, during and following oxygen therapy. If a pulse oximeter is available, it should be used. ${ }^{2}$ However, usually only dental practices that administer sedation will have a

pulse oximeter.

\section{OXYGEN FACE MASK WITH OXYGEN RESERVOIR}

An oxygen face mask with oxygen reservoir (sometimes called an oxygen non-rebreathe mask) (Fig. 1) has a one-way valve that diverts the oxygen flow into the reservoir bag during expiration; the contents of the reservoir bag together with the high flow oxygen (15 litres per minute), results in minimal entrainment of air and an inspired oxygen concentration of approximately $90 \% .{ }^{1}$ The valve also prevents the patient's exhaled gases from entering the reservoir bag. The use of the oxygen reservoir bag helps to increase the inspired oxygen concentration by preventing oxygen loss during inspiration.

To ensure the mask is functioning correctly and is effectively used, it is important to follow the manufacturer's recommendations for simple basic checks before use. ${ }^{6}$

\section{PORTABLE OXYGEN CYLINDER}

Portable oxygen cylinders are black with white shoulders. ${ }^{7}$ There are many different types of portable cylinders available. A commonly used one is featured in Figure 2. This cylinder has:

- A regulator integrated with the cylinder valve

- A gauge that shows 'live' contents at all times, even when the cylinder is turned off

- Simple on-off handwheel (no tools needed)
- Click-stop flow control knob showing flow in litres/min, ranging from 1 to 15 litres per minute

- Sufficient capacity to last approximately 30 minutes at highest flow rate of 15 litres/min. ${ }^{8}$

Always follow the manufacturer's recommendations when using an oxygen cylinder. Before using the oxygen cylinder, as displayed in Figure 2:

- If necessary, remove the protective cap

- Attach the oxygen tubing and mask to the fir tree outlet (the oxygen outlet) of the cylinder

- Ensure the flow control knob on the top of the cylinder is set at ' 0 '

- Open the valve slowly by turning the grey or black handwheel anti-clockwise two revolutions

- Turn the flow control knob clockwise to select the required flow rate. At each full 'click' a different flow rate setting will be revealed in the 'window' of the knob. The correct flow rate setting must be fully visible in the window

- Select flow rate as per training received

- Check for a flow of gas through the mask before use. ${ }^{8}$

After using the oxygen cylinder, as displayed in Figure 2:

- Turn off cylinder using the grey or black handwheel by turning it clockwise

- Remove tubing and mask from the fir tree outlet and allow residual oxygen in the regulator to vent

- Turn flow control knob to '0'

- Check the cylinder contents gauge to ensure adequate supply for next administration. $^{8}$

\section{GUIDELINES ON THE ADMINISTRATION OF OXYGEN}

The British Thoracic Society (BTS) ${ }^{9}$ has issued detailed guidance on emergency oxygen therapy in adults. The guidance relating to the use of high flow oxygen has caused some concern and confusion regarding its safety. It is emphatically clear that in any critically ill patient the initial administration of high flow oxygen (15 litres per minute) is the correct course of action. When oxygen saturation levels can be accurately measured using a pulse oximeter, the amount of oxygen administered can be titrated accordingly.

PROCEDURE FOR ADMINISTRATION OF OXYGEN USING A NON-REBREATHE MASK

- Assess and treat the patient following the 'ABCDE' (Airway, Breathing,
Circulation, Disability and Exposure) approach; call for help from colleagues. It may be necessary to call 999 for an ambulance

- Explain the procedure to the patient

- If available, attach pulse oximetry (usually only dental practices that administer sedation will have a pulse oximeter) to guide oxygen therapy

- Ensure the patient is in an appropriate position for example, if breathless, the patient will usually prefer to be in an upright position

- Prepare the oxygen cylinder and attach the oxygen tubing (see above)

- Select an oxygen flow rate of 15 litres/ $\min$

- Occlude the valve between the mask and the oxygen reservoir bag and check that the reservoir bag is filling up. Remove the finger

- Squeeze the oxygen reservoir bag to check the patency of the valve between the mask and the reservoir bag. If the valve is working correctly it will be possible to empty the reservoir bag (if the reservoir bag doesn't empty discard it and select another mask)

- Again occlude the valve between the mask and the oxygen reservoir bag, allowing the reservoir bag to fill up

- Place the mask with a filled oxygen reservoir bag on the patient's face, ensuring a tight fit

- Reassure the patient

- Closely monitor the patent's vital signs. In particular, assess the patient's response to the oxygen therapy for example, respiratory rate, mechanics of breathing, colour, oxygen saturation levels (if pulse oximetry available), level of consciousness.

- Discontinue/reduce the inspired oxygen concentration as appropriate following advice from a suitably qualified dental practitioner

- Await the arrival of the ambulance.

\section{CHRONIC OBSTRUCTIVE PULMONARY DISEASE (COPD) PATIENTS}

Although oxygen should be administered with extreme caution in patients with COPD ${ }^{8}$ in critical illness high flow oxygen as described above should be administered. ${ }^{1}$

\section{CARE, HANDLING AND STORAGE OF OXYGEN CYLINDERS}

The Medicines and Healthcare products Regulatory Agency (MHRA) ${ }^{10}$ has issued guidance on the care and handling of oxygen cylinders and their regulators. It recommends that healthcare staff should: 
- Be fully trained in the use of oxygen cylinders

- Be aware of all the related risks such as fire and manual handling

- Carry out full checks on oxygen cylinders and their regulators before each use, ensuring that they contain enough oxygen for the required therapy

- Check the label on the oxygen cylinder ensuring it is not out-of-date

- Ensure their hands are clean before handling an oxygen cylinder; there is a risk of combustion from oils and grease. It is also important to ensure their hands are adequately dried after the use of alcohol gel

- Ensure that the oxygen cylinder outlet and oxygen regulator inlet are clean before attaching a regulator

- Always open the cylinder slowly and check for leaks. Close cylinder valves when not in use

- Handle oxygen cylinders with care. If the cylinder is dropped or knocked in use it must be checked before further use; cylinders with integral valves should be returned to the supplier; separate regulators should be sent to the service department for inspection.

The MHRA ${ }^{10}$ recommends that oxygen cylinders should be stored in a secure area that is well ventilated, clean and dry, as well as being free from any sources of ignition such as patients/staff smoking or machinery. BOC Medical ${ }^{9}$ advises that it is important to:

- Keep the oxygen cylinder away from naked flames and sources of heat. Oxygen is a non-flammable gas, but it does strongly support combustion
- Ensure the oxygen cylinders are stored in a safe and secure area where they cannot fall over and cause injury

- Ensure the oxygen cylinder is stored in a well-ventilated area

- Never use excessive force when opening or closing the cylinder using the grey or black handwheel

- Not paint the cylinders as all labels and markings must remain clearly visible

- Refrain from using oil or grease (or any oil-based products, which includes hand creams) in the vicinity of the oxygen cylinder. High velocity oxygen and oil/grease could cause spontaneous combustion. ${ }^{9}$

Defective oxygen cylinders should be reported to the Defective Medicines Reporting Centre (DMRC) and defective detachable regulators to the Adverse Incident Centre (AIC), both at the MHRA (www.mhra.gov.uk).

\section{CONCLUSION}

Critically ill patients should receive high flow oxygen. Dental practitioners should understand when and how to safely and effectively administer oxygen. An overview of the hazards associated with the use and storage of oxygen has been provided together with the procedure for administering it.

\section{GLOSSARY}

- Dyspnoea: difficult or laboured breathing (derived from Latin and Greek words: dus - bad or difficult, pnoe -breathing)

- Cyanosis: blue discolouration of the skin due to poor circulation or inadequate oxygenation of the blood (derived from the Greek word kuanosis - blueness)

- Haemoglobin: a red protein containing iron, responsible for transporting oxygen in the blood; the normal haemoglobin (HB) count in men is 13-18 g per $100 \mathrm{ml}$ and in women is $12-16 \mathrm{~g} / 100 \mathrm{ml}$

- Anaemia: condition characterised by inadequate red blood cells and/or haemoglobin in the blood; in men $<13 \mathrm{~g}$ per $100 \mathrm{ml}$ of blood and in women $<12 \mathrm{~g}$ per $100 \mathrm{ml}$

- Hypoxia: deficient oxygenation of the tissues (derived from the Greek word hupo - under and oxygen)

- Hypoxaemia: fall in concentration of oxygen in the arterial blood

- Pulse oximetry: measurement of oxygen saturation of haemoglobin; the normal oxygen saturation of arterial blood is $94 \%$ or higher.

1. Resuscitation Council UK. Medical emergencies and resuscitation standards for clinical practice and training for dental practitioners and dental care professionals in general dental practice. London: Resuscitation Council UK, 2012.

2. National Patient Safety Agency. Oxygen safety in hospitals. NPSA, 2009.

3. Jevon P. Practical procedures: administering oxygen Dental Nursing 2009; 5: 615-617.

4. British Medical Association, Royal Pharmaceutical Society of Great Britain. British national formulary 65. London: BMA, 2013.

5. Higgins D. Oxygen therapy. Nurs Times 2005; 101 : 30-31.

6. Intersurgical. Non-rebreathing mask product literature. Wokingham: Intersurgical, 2003.

7. Marcovitch H. Black's medical dictionary. 41st ed. London: A \& C Black, 2005.

8. BOC Medical. Instructions for using a CD oxygen cylinder.

9. British Thoracic Society. Emergency oxygen use in adult patients. London: BTS, 2008. Online guidelines available at http://www.brit-thoracic. org.uk/guidelines/emergency-oxygen-use-in-adultpatients.aspx (accessed October 2013).

10. Medicines and Healthcare Products Regulation Agency. Top tips on care and handling of oxygen cylinders and their regulators. London: MHRA, 2008. 